International Journal of Difference Equations (IJDE).

ISSN 0973-6069, Volume 15, Number 1, (2020). 71-82

(C) Research India Publications

https://dx.doi.org/10.37622/IJDE/15.1.2020.71-82

\title{
Variant of Laplace Transform Represented by a Logarithmic Function
}

\author{
Hwajoon Kim, Seunghag Beak, and Jinho Rho \\ Kyungdong University, 11458, Kyungdong Univ. Rd. 27, Yangju, Gyeonggi, S. Korea.
}

\begin{abstract}
In this study, we consider a variant of a generalized Laplace transform represented by a logarithmic function. The tool of the proposed research is the substitution to logarithmic function. In addition, we investigated the existence and uniqueness theorem on the variant of Laplace transform. The obtained result has a strong point in existence theorem because of its simplicity. As a result, the proposed theorem does not need the exponential function in the condition of growth restriction. The main objective of this paper is to apply the result in a new generalized integral transform.
\end{abstract}

AMS Subject Classification: 44A05, 35A22

Key Words and Phrases: generalized Laplace transform, variant of transform, existence theorem

\section{INTRODUCTION}

The method of Laplace transform[10] is a tool to solve ordinary differential equations(ODEs), a way to find analytical solutions of partial differential equations(PDEs), and it is a fundamental thing among many integral transforms[1, $3-4,15]$. Therefore, this manuscript focused on a variant of the Laplace transform for appropriate research scope. Owing to simplicity, this paper does not cover a generalized Laplace transform, because the extension to it is not difficult. 
The intrinsic structure of a generalized Laplace transform[6, 14] is given by

$$
(G £)(f)=u^{\alpha} \int_{0}^{\infty} e^{-\frac{t}{u}} f(t) d t
$$

and the Laplace transform has a value of $\alpha=0$ for $(G £)(f)(=F(u))$ is a generalized Laplace transform. The alternate form of the generalized Laplace transform is given by

$$
(G £)(f)=u^{\beta} \int_{0}^{\infty} e^{-t} f(u t) d t
$$

and if $\beta=1,(G £)(f)$ corresponds to the Laplace transform. Additionally, by substitution $t=\ln x$, the Laplace transform $£(f)$ can be changed to

$$
\int_{1}^{\infty} f(\ln x) \frac{1}{x^{s+1}} d x
$$

As this form is just a variant of the Laplace transform, we can use the Laplace's formula without substituting $t=\ln x$. Additionally, owing to its simplicity, this variant has a strong point in existence theorem which does not need the exponential function in the condition of growth restriction. Let us denote this form by $L(f)$, which is Gothic type, i.e.,

$$
L(f)=\int_{1}^{\infty} f(\ln x) \frac{1}{x^{s+1}} d x .
$$

Hence, we can naturalize the generalized Laplace transform as

$$
(G L)(f)=s^{-\alpha} \int_{1}^{\infty} f(\ln x) \frac{1}{x^{s+1}} d x
$$

and the details are given in the next section.

As a modified Laplace transform, Sumudu transform, Elzaki one, Mohand one, and natural one have been introduced[1, 3-4, 11, 15]. On the one hand, Jhanthanam et al. found the generalized solutions of the third-order Cauchy-Euler equation in the space of right-sided distributions using Laplace transform[5], investigated the form and properties of an integral transform that has strength in transform of integral[8], and studied a new class of Laplace-type integrals involving generalized hypergeometric functions[9]. A research related to exact solutions of some Schrodinger equations has investigated[2]. Moreover, for further developments of this research, a qualitative analysis could be also performed for partial differential equations driven by a combination of differential operators of different nature (e.g., (p,2)-equations)[13].

On the other hand, in pedagogy, the Spearman's rank correlation coefficient plays an important role in checking the correlation between two variables, such as finding the 
correlation between the class ranking of a third grader and that of a fourth grader[12]. The coefficient comprises five steps; very weak, weak, moderate, strong, and very strong, with each parameter being a nonparametric measure of the rank correlation. This coefficient can be applied in case of a monotonic relationship between two variables. It is clear that the utilization of the monotonicity is higher than that of linearity. We studied the Spearman rank correlation coefficient represented by a variant of the Laplace transform, and this method can be applied in case of large sample size.

The following results are obtained;

(1) If a function $f$ is integrable, then

$$
\text { (b)L(f } \left.f^{(n)}\right)=s^{n} L(f)-\sum_{k=1}^{n} s^{n-k} f^{(k-1)}(0) \text {, }
$$

is valid for $f^{(n)}$ is the $n$-th derivative of $f$.

(2) Linearity, existence, and uniqueness theorem on a variant of the Laplace transform;

(a) Let $L(t)$ be the aforementioned variant of the Laplace transform. Then $L(t)$ is a linear operation.

(b) If $f(x)$ is defined, piecewise continuous on every finite interval on the semi-axis $x \geq 0$, and satisfies

$$
|f(\ln x)| \leq M x^{k}
$$

for all $x \geq 0$ and some constants $M$ and $k$, then the variant of the Laplace transform $L(f)$ exists for all $s>k$.

(c) If the variant of the Laplace transform of a given function exists, then it is uniquely determined.

\section{KERNELS IN INTEGRAL TRANSFORMS AND THE NECESSITY OF THIS RESEARCH}

Integral transforms can be extensively utilized in computed tomography(CT) scan or magnetic resonance imaging(MRI). Typically, projection data are obtained by integral transform, and an image using inverse transform is produced. Additionally, the concept of convolution in integral transform can be linked to the convolution of artificial intelligence(AI). In general, integral transforms are in the form of

$$
\int_{0}^{\infty} k(s, t) f(t) d t
$$


where $k$ is the kernel. It is well-known that the Laplace transform has the kernel $k(s, t)=e^{-s t}$. Since the exponential function has the property of $e^{-s t}$ converging to 0 when $t$ approaches $\infty$, an exponential function is, therefore, normally used as the kernel in integral transforms[6], where $s>0$. A form of a generalized Laplace-typed integral transform is mentioned in the introduction as

$$
(G £)(f)=u^{\alpha} \int_{0}^{\infty} e^{-\frac{t}{u}} f(t) d t,
$$

for $(G £)(f)=F(u)$. Here, the Laplace transform has the value $\alpha=0$. If $a=-1$, then it becomes sumudu transform. If $a=1(a=-2)$, then it becomes Elzaki transform (Mohand one), respectively. An alternative representation of this is

$$
(G £)(f)=u^{\beta} \int_{0}^{\infty} e^{-t} f(u t) d t,
$$

if $\beta=1$, then $(G £)(f)$ corresponds to the Laplace transform. Similarly, if $\beta=0$, $\beta=2$, and $\beta=-1$, then it corresponds to the Sumudu transform, Elzaki transform, and Mohand transform[11], respectively.

The simplest form of a generalized Laplace-typed transform is in the form of

$$
F(s)=£(f)=s^{\gamma} \int_{0}^{\infty} e^{-s t} f(t) d t .
$$

In this form, the Laplace transform, Sumudu transform, Elzaki transform, and Mohand transform have the value of $\gamma$ as $0,1,-1$, and 2, respectively. A simple way to derive the Sumudu transform is to multiply the Laplace transform by s. Similarly, it can be multiplied multiply by $s^{-1}$ to obtain the Elzaki transform, and multiplied by $s^{2}$ to obtain the Mohand transform. The natural transform[1] can be obtained by substituting $f(t)$ with $f(u t)$. Hence, it can be observed that slight changes in the kernel can bring about significant changes in the transform. By substituting $t=\ln x$, the Laplace-typed transform can be generalized as

$$
(G L)(f)=s^{-\delta} \int_{1}^{\infty} f(\ln x) \frac{1}{x^{s+1}} d x
$$

The Laplace transform has the value $\delta=0$, hence, the form of the Laplace transform can be represented as

$$
L(f)=\int_{1}^{\infty} f(\ln x) \frac{1}{x^{s+1}} d x=\int_{1}^{\infty} f(\ln x) x^{-s-1} d x .
$$

Additionally, there is a Mellin transform[3] of the form

$$
\int_{0}^{\infty} f(x) x^{s-1} d x
$$


similar to (5). However, since the interval of integration and the power of kernel are different, it can be considered as a completely different form. Consequentially, it is not a new transform but a variant of Laplace transform. However, the proposed variant provides computational aid in the study of generalized transforms.

The main objective of this paper is to apply the result in a new generalized integral transform. The new generalized transform will be described in a further study.

Table 1: Table of Laplace-typed integral transform $G_{\alpha}[4]$

\begin{tabular}{ccccc}
\hline & $\mathbf{L}$ & $\mathbf{S}$ & $\mathbf{E}$ & $\mathbf{M}$ \\
\hline$\alpha$ & 0 & -1 & 1 & -2 \\
$\beta$ & 1 & 0 & 2 & -1 \\
$\gamma$ & 0 & 1 & -1 & 2 \\
\hline
\end{tabular}

where, L, S, E, and $\mathrm{M}$ are Laplace, Sumudu, Elzaki, and Mohand transform, respectively.

\section{VARIANT OF LAPLACE TRANSFORM REPRESENTED BY A LOGARITHMIC FUNCTION}

In introduction, we have mentioned that we can use the Laplace's formula without changing it in the calculation of the aforementioned variant of the Laplace transform. Let us check about this. For example, if $f(t)=f(\ln x)=1$, then $L(1)=$ $\int_{1}^{\infty} x^{-(s+1)} d x=1 / s=£(1)$. Similarly, if $f(t)=f(\ln x)=t$,

$$
L(t)=\int_{1}^{\infty} x^{-(s+1)} \ln x d x .
$$

Putting $\ln x=t$, we have

$$
L(t)=\int_{0}^{\infty} e^{-t(s+1)} t e^{t} d t=\int_{0}^{\infty} e^{-t s} t d t .
$$

By the integration by part, we obtain the solution $L(t)=1 / s^{2}$. Let us check one more thing. If $f(t)=e^{a t}$,

$$
L\left(e^{a t}\right)=\int_{1}^{\infty} x^{-(s+1)} e^{a \ln x} d x=\int_{1}^{\infty} x^{a-s-1} d x=\frac{1}{s-a}
$$

for $s>a$. More exactly,

$$
L(f)=\int_{1}^{\infty} f(\ln x) x^{-s-1} d x=\int_{0}^{\infty} e^{-t(s+1)} f(t) e^{t} d t=£(f)
$$


for $x=e^{t}$. Consequently, since this form is a variant of the Laplace transform, we can use the formulas of the Laplace transform as it is. The following statement follows;

Theorem 1. If a function $f$ is integrable, then

$$
L\left(f^{(n)}\right)=s^{n} L(f)-\sum_{k=1}^{n} s^{n-k} f^{(k-1)}(0),
$$

is valid for $f^{(n)}$ is the $n$-th derivative of $f$.

Proof. Note that $L(t)=\int_{1}^{\infty} x^{-s-1} f(\ln x) d x$, and let us approach the proof by induction. In case of $n=1$,

$$
L\left(f^{\prime}\right)=\int_{1}^{\infty} x^{-s-1} f^{\prime}(\ln x) d x
$$

Putting $t=\ln x$, we have

$$
\begin{gathered}
L\left(f^{\prime}\right)=\int_{0}^{\infty} e^{-t(s+1)} f^{\prime}(t) e^{t} d t \\
=\int_{0}^{\infty} e^{-t s} f^{\prime}(t) d t \\
=\left[e^{-t s} f(t)\right]_{0}^{\infty}+s \int_{0}^{\infty} e^{-t s} f(t) d t=-f(0)+s L(f)
\end{gathered}
$$

because of $d x=e^{t} d t$. This is the same as the value from the second statement.

Next, let us suppose that $n=m$ is valid for some $m$. Thus,

$$
L\left(f^{(m)}\right)=s^{m} L(f)-\sum_{k=1}^{m} s^{m-k} f^{(k-1)}(0)
$$

holds for $f^{(m)}$ is the $m$-th derivative of $f$. Let us show that

$$
L\left(f^{(m+1)}\right)=s^{m+1} L(f)-\sum_{k=1}^{m+1} s^{m+1-k} f^{(k-1)}(0) .
$$

$L\left(f^{(m+1)}\right)$ can be represented as

$$
\begin{gathered}
L\left(f^{(m+1)}\right)=\int_{1}^{\infty} x^{-s-1} f^{(m+1)}(\ln x) d x \\
=\int_{0}^{\infty} e^{-t s} f^{(m+1)}(t) d t
\end{gathered}
$$


by the same way with the case of $n=1$. Integration by parts gives

$$
\begin{gathered}
L\left(f^{(m+1)}\right)=\left[e^{-t s} f^{(m)}(t)\right]_{0}^{\infty}+s \int_{0}^{\infty} e^{-t s} f^{(m)}(t) d t \\
=-f^{(m)}(0)+s L\left(f^{(m)}\right) \\
=-f^{(m)}(0)+s\left[s^{m} L(f)-\sum_{k=1}^{m} s^{m-k} f^{(k-1)}(0)\right] \\
=-f^{(m)}(0)+s^{m+1} L(f)-\sum_{k=1}^{m} s^{m+1-k} f^{(k-1)}(0) \\
=s^{m+1} L(f)-\sum_{k=1}^{m+1} s^{m+1-k} f^{(k-1)}(0)=(*) .
\end{gathered}
$$

Hence, this theorem is valid at an arbitrary natural number $n$.

The followings are the direct results of theorem 1;

$$
\begin{gathered}
L\left(f^{\prime}\right)=s L(f)-f(0) \\
L\left(f^{\prime \prime}\right)=s^{2} L(f)-s f(0)-f^{\prime}(0)
\end{gathered}
$$

for $F=L(f)$.

Let us see a quite familiar example on semi-infinite string.

Example 1. Let us consider an elastic string which is fastened at the ends $x=0$ and $x=\pi$, and assume that its initial deflection $w(x, 0)$ and initial velocity $w_{t}(x, 0)$ are both 0 . Then the equation has the form of

$$
w_{t t}=c^{2} w_{x x}
$$

subject to $w(x, 0)=0, w_{t}(x, 0)=0$, and

$$
\lim _{x \rightarrow \infty} w(x, t)=0,
$$

and let us assume that the left end of the string is moved in a single sine wave

$$
w(0, t)=f(t)= \begin{cases}\sin 2 t & \text { if } 0 \leq t \leq \pi \\ 0 & \text { otherwise }\end{cases}
$$


Solution. Taking Laplace transform on both sides, from theorem 1, we have

$$
s^{2} W-s w(0, t)-w_{t}(x .0)=s^{2} W=c^{2} \frac{d^{2} W}{d x^{2}}
$$

for $W(x, s)=L(w(x, t))$. Consider $\mathrm{W}(\mathrm{x}, \mathrm{s})$ as a function of $x$. Since this equation is regarded as a homogeneous linear ODE with constant coefficients, a general solution can be written as

$$
W(x, s)=A(s) e^{s x / c}+B(s) e^{-s x / c} .
$$

From the boundary conditions, $W(0, s)=F(s)$ and

$$
\lim _{x \rightarrow \infty} W(x, s)=\lim _{x \rightarrow \infty} \int_{1}^{\infty} x^{-s-1} f(\ln x) d x=0,
$$

because of $s>0$. Thus, $A(s)=0$ follows, so that

$$
W(x, s)=F(s) e^{-s x / c}
$$

because of $W(0, s)=B(s)=F(s)$. The second shifting theorem

$$
L\{f(t-a) u(t-a)\}=e^{-a s} F(s)
$$

gives

$$
w(x, t)=f\left(t-\frac{x}{c}\right) u\left(t-\frac{x}{c}\right)=\sin 2\left(t-\frac{x}{c}\right)
$$

if $x / c<t<x / c+\pi$.

In the above integral, we note that $u$ is the unit step function. Of course, this proof was made on the premise that integration and differentiation could be interchanged. Nevertheless, the solution

satisfies the given equation

$$
w(x, t)=\sin 2\left(t-\frac{x}{c}\right)
$$

$$
w_{t t}=c^{2} w_{x x}
$$

because

and

$$
w_{t}=2 \cos 2\left(t-\frac{x}{c}\right), w_{x}=-\frac{2}{c} \cos 2\left(t-\frac{x}{c}\right)
$$

$$
w_{t t}=-4 \sin 2\left(t-\frac{x}{c}\right), w_{x x}=-\frac{4}{c^{2}} \sin 2\left(\left(t-\frac{x}{c}\right) .\right.
$$

The definition of the Laplace transform by a logarithm function has a different environment for the existence theorem. In other word, the traditional Laplace transform needs a growth restriction represented by an exponential function, whereas the aforementioned variant of the Laplace transform does not need the exponential function. Let us see the following result. 
Theorem 2. (Linearity, existence, and uniqueness theorem on a variant of the Laplace transform)

(1) Let $L(t)$ be the aforementioned variant of the Laplace transform. Then $L(t)$ is a linear operation.

(2) If $f(x)$ is defined, piecewise continuous on every finite interval on the semi-axis $x \geq 0$, and satisfies

$$
|f(\ln x)| \leq M x^{k}
$$

for all $x \geq 0$ and some constants $M$ and $k$, then the variant of the Laplace transform $L(f)$ exists for all $s>k$.

(3) If the variant of the Laplace transform of a given function exists, then it is uniquely determined.

Proof. (1) $L\{a f(x)+b g(x)\}=\int_{1}^{\infty} x^{-s-1}\{a f(\ln x)+b g(\ln x)\} d x=a L(f(x))+$ $b L(g(x))$.

(2) Note that piecewise continuity of $f(x)$ ensures integrability of $x^{-s-1} f(\ln x)$ on any finite interval.

$$
\begin{array}{rl}
|L(f)|=\mid \int_{1}^{\infty} x^{-s-1} & f(\ln x) d x\left|\leq \int_{1}^{\infty} x^{-s-1}\right| f(\ln x) \mid d x \\
\leq & \int_{1}^{\infty} x^{-s-1} M x^{k} d x \\
= & M \int_{1}^{\infty} x^{k-s-1} d x \\
& =M\left[\frac{1}{k-s} x^{k-s}\right]_{1}^{\infty} \\
& =\frac{M}{s-k}<\infty
\end{array}
$$

(3) Assume that $L(f)$ exists by $L\left(f_{1}\right)$ and $L\left(f_{2}\right)$ both. If $L\left(f_{1}\right) \neq L\left(f_{2}\right)$ for $f_{1}=f_{2}$, then

$$
\begin{gathered}
L\left(f_{1}\right)-L\left(f_{2}\right)=\int_{1}^{\infty} x^{-s-1} f_{1}(\ln x) d x-\int_{1}^{\infty} x^{-s-1} f_{2}(\ln x) d x \\
=\int_{1}^{\infty} x^{-s-1}\left[f_{1}(\ln x)-f_{2}(\ln x)\right] d x \\
=\int_{1}^{\infty} x^{-s-1}\left(f_{1}-f_{2}\right)(\ln x) d x \\
=L\left(f_{1}-f_{2}\right)=0 .
\end{gathered}
$$


This is a contradiction on $L\left(f_{1}\right) \neq L\left(f_{2}\right)$, and hence the transform is uniquely determined. Conversely, if two functions $f_{1}$ and $f_{2}$ have the same transform(i.e. $\left.L\left(f_{1}\right)=L\left(f_{2}\right)\right)$, then

$$
L\left(f_{1}\right)-L\left(f_{2}\right)=\int_{1}^{\infty} x^{-s-1}\left(f_{1}-f_{2}\right)(\ln x) d x=0,
$$

and so $f_{1}=f_{2}$ a.e.. Hence $f_{1}=f_{2}$ excepting for the set of measure zero.

Since the variant of the generalized Laplace transform $G £(f)$ represented by a logarithmic function can be represented by the similar way, we will not deal with the details.

Table 2: Table of Laplace-typed integral transform $G_{\alpha}[14]$

\begin{tabular}{ccc}
\hline & $f(t)$ & $G_{\alpha}(f)$ \\
\hline 1 & 1 & $u^{\alpha+1}$ \\
2 & $\mathrm{t}$ & $u^{\alpha+2}$ \\
3 & $t^{n}$ & $n ! \cdot u^{n+\alpha+1}$ \\
4 & $e^{a t}$ & $\frac{u^{\alpha+1}}{1-a u}$ \\
5 & $\sin a t$ & $\frac{a u^{\alpha+2}}{1+u^{2} a^{2}}$ \\
6 & $\cos a t$ & $\frac{u^{\alpha+1}}{1+u^{2} a^{2}}$ \\
7 & $\sinh a t$ & $\frac{a u^{\alpha+2}}{1-u^{2} a^{2}}$ \\
8 & $\cosh a t$ & $\frac{u^{\alpha+1}}{1-u^{2} a^{2}}$ \\
9 & $e^{a t} \cos b t$ & $\frac{u^{\alpha}\left(\frac{1}{u}-a\right)}{\left(\frac{1}{u}-a\right)^{2}+b^{2}}$ \\
10 & $e^{a t} \sin b t$ & $\frac{b u^{\alpha}}{\left(\frac{1}{u}-a\right)^{2}+b^{2}}$ \\
\hline
\end{tabular}

On the other hand, the Spearman rank-order correlation coefficient $r_{s}($ or $\rho$ ) has a form of

$$
r_{s}=1-\frac{6 \sum d^{2}}{n(n-1)(n+1)},
$$

where $d$ is the difference between the two ranks of each observation. Taking the variant of the Laplace transform, we have

$$
L\left(r_{s}\right)=6 \sum d^{2}+\frac{1}{s}-36 \sum d^{2} \frac{1}{s^{2}-6}
$$

because of

$$
L(1)=\int_{1}^{\infty} x^{-s-1} \cdot 1 d x=1 / s=£(1) .
$$

Taking the inverse transform, we can obtain the coefficient $r_{s}$. 
Conflict of interest The author declare that there is no conflict of interest regarding the publication of this paper.

Acknowledgements This research was supported by Kyungdong University Research Fund, 2020.

\section{REFERENCES}

[1] F. B. M. Belgacem and R.Silambarasan, Theory of natural transform, Mathematics in Engineering, Science and Aerospace, 3 (2012), 105-135.

[2] Y. Benia, M. Ruggieri and A. Scapellato, Exact Solutions for a Modified Schrodinger Equation, Mathematic, 7 (2019), 908

[3] Bertrand, J., Bertrand, P., and Ovarlez J.P., The Mellin Transform, The Transforms and Applications, Handbook (A.D. Poularkas, ed.). CRC Press, Boca Raton, FL, 1996.

[4] T. M. Elzaki, S. M. Ezaki and E. M. A. Hilal, ELzaki and Sumudu Transform for Solving some Differential Equations, Glob. J. of Pure \& Appl. Math., 8 (2012), 167-173.

[5] Jhanthanam S, Nonlaopon K, Orankitjaroen S. Generalized Solutions of the Third-Order Cauchy-Euler Equation in the Space of Right-Sided Distributions via Laplace Transform, Mathematics, 7 (2019), 376.

[6] $\mathrm{Hj}$. Kim, The intrinsic structure and properties of Laplace-typed integral transforms, Mathematical Problem in Engineering, 2017 (2017), 1-8.

[7] Hj. Kim, The solution of the heat equation without boundary conditions, Dynamic Systems and Applications, 27 (2018), 653-662.

[8] $\mathrm{Hj}$. Kim, On the form and properties of an integral transform with strength in integral transforms, Far East. J. Math. Sci. 102 (2017), 2831-2844.

[9] Koepf, Wolfram, Insuk Kim, and Arjun K. Rathie, On a New Class of Laplace-Type Integrals Involving Generalized Hypergeometric Functions, Axioms, 8, (2019), P87.

[10] E. Kreyszig, Advanced Engineering Mathematics, Wiley, Singapore, 2013.

[11] Mohand M. Abdelrahim Mahgoub, The New Integral Transform 'Mohand Transform', Advances in Theoretical and Applied Mathematics 12, (2017), 113-120.

[12] Yongjoo Lee and Hwajoon Kim, The Spearman rank-order correlation coefficient represented by Laplace transform, JP J of heat and mass transfer, 18, (2019), 363-372. 
[13] N. S. Papageorgiou and A. Scapellato, Positive solutions for $(p, 2)$-equations with superlinear reaction and a concave boundary term, Electron., J. Qual. Theory Differ. Equ, 4, (2020), 1 ?19

[14] S. Supaknaree, K. Nonlaopon, and Hj. Kim, Further properties of Laplace-type integral transforms, Dynamic Systems and Applications, 28 (2019), 195-215.

[15] G. K. Watugula, Sumudu Transform: a new integral transform to solve differential equations and control engineering problems, Integrated Education, 24 (1993), $35-43$. 